\title{
Design and Development of a Digital Stethoscope for Cardiac Murmur
}

\author{
Kuldeep Singh \\ Centre for Development of Advanced Computing, \\ Mohali, Punjab, India
}

\author{
Preeti Abrol \\ Centre for Development of Advanced Computing, \\ Mohali, Punjab, India
}

\begin{abstract}
The stethoscope has been used for many years, and it has been very effective to diagnose certain pulmonologic and cardiologic sounds. From many years healthcare professionals would listen quietly to patients internal organs so they could diagnose from specific sounds of such internal organs. The sound level of an acoustic stethoscope is very low, making heart diagnosis more difficult. In this paper, an electronic stethoscope, which is based on embedded processors, is designed to fulfill the shortages from the auscultation. It converts acoustic sound into electrical signal and this signal is amplified and heard on speakers, so there is no need of headphone for listening heart sounds. Heart Beats per Minute (HBM) is displayed on LCD. The cardiac sound waveform is displayed using MATLAB on a PC via UART interface of the microcontroller. It serves as a platform for potential Computer Aided Diagnosis (CAD) applications for the detection of cardiac murmurs which offers signal amplification and also added functionalities like storage, analysis and visual representation of sound signals.
\end{abstract}

\section{Keywords}

Heart sounds, Digital stethoscope, ATmega16

microcontroller

\section{INTRODUCTION}

The Cardiac auscultation is one of the most useful investigative tools that the physician can use at the bedside to detect alterations in cardiovascular anatomy and physiology. Typically, heart sounds and murmurs are of relatively low intensity and are band limited to about 10-120 $\mathrm{Hz}$. Meanwhile, speech signal is perceptible to the human hearing. Therefore, auscultation with an acoustic stethoscope is quite difficult. Only a s mall proportion of cardiovascular sound energy is audible by the human ear. The problem with acoustic stethoscopes is that the sound level is low, making diagnosis more difficult. The objective is to develop a AVR based Digital Stethoscope to capture heart sounds and diagnose them with the help of display and analytic tools. [1]

The proposed design of the system includes: ATmega16 microcontroller, graphic LCD, memory and MATLAB interface. The objective is to develop a technique which makes a clear distinction between normal heart sounds and heart murmurs. The data can be analyzed by using analytic tool such as MATLAB.

The paper is organized in five sections.

Section 2 explains the problem definition. Section 3 contains the block diagram and description of proposed system, Section 4 contains results for proposed design and Section 5 ends with conclusion.

\section{PROBLEM DEFINATION}

Heart patients are needed to be monitored continuously so that diagnosis can be made correctly and preventive measures can be taken. Acoustic stethoscope used for auscultation are mainly consisting of diagnosis on the basis of hearing cardiac sound thus, it is difficult to differentiate between cardiac sound and suspicious murmur. Heart murmurs may go unnoticed during routine check-ups due to low intensity, the quality of the device used, and the severity of the condition. A digital stethoscope can be used to help physicians in analyzing cardiac signals in real time during auscultation. This helps to reduce the risks and take preventive steps.

\section{SYSTEM DESCRIPTION}

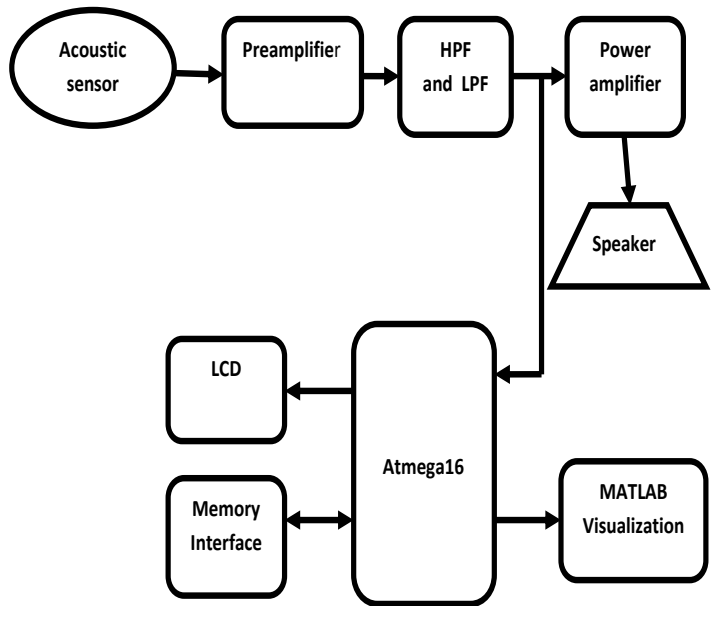

Figure 1. Block diagram of the proposed system

The proposed system consists of the following hardware components: 1) ATmega16 microcontroller 2) Microphone Bias and Amplifier Circuit 3) LCD 4) Serial EEPROM 5) MATLAB interface. These modules have been shown in Figure 1.

\subsection{MCU (ATmega16)}

The ATmega16 is 8-bit microcontroller based on the AVR enhanced RISC architecture. It is a low-power CMOS device. By executing powerful instructions in a single machine cycle, the ATmega16 achieves throughputs approaching 1 MIPS per $\mathrm{MHz}$ allowing the system designed to optimize power consumption versus processing speed. It can work at maximum frequency of $16 \mathrm{MHz}$. 
There are ATmega16 features that described below:

- High-performance

- Low-power Atmel AVR 8-bit Microcontroller

- $\quad$ Advanced RISC Architecture

$>131$ Powerful Instructions - mostly Singlemachine Cycle Execution

$>$ General Purpose Registers (32*8)

- Non-volatized and High Endurance Memory segments

$>16$ Kilobytes of In-System Self-programmable Flash program memory

> 512 Bytes EEPROM

$>1 \mathrm{~K}$ byte Internal SRAM

\subsection{Acoustic Sensor}

The stethoscope acoustic sensor is an integral hardware component of the system. It consists of a diaphragm on which the piezoelectric substance is mounted, a piezoelectric element on which electrode is mounted which is retained in the center of a frame having a horn-shape inward, a pair of membrane capable of vibrating which is placed opposite ends of the frame to form a cell, an electrical insulating liquid is hermetically enclosed in the cell, and leads are drawn out from the electrodes to the outside.

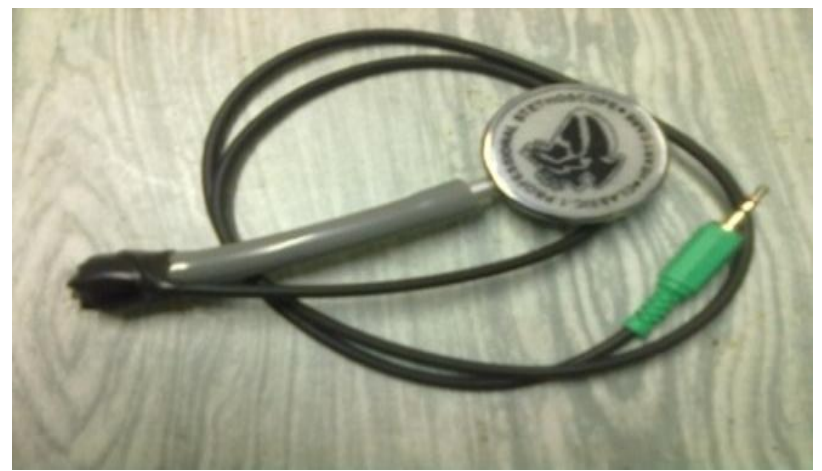

Figure 2. Stethoscope acoustic sensor

The quality of the sensor directly affects the quality of the real-time audio output as well as all analyses performed on the measured waveforms. The sensor includes a standard stethoscope chest piece to amplify acoustic signals and an electret condenser microphone to convert the amplified signals to electrical waveforms. The microphone is placed within a rubber tube at a distance of $18 \mathrm{~cm}$ from chest piece base.

\subsection{Microphone Bias and Amplifier Circuit}

The microphone in the acoustic sensor needed to be biased in order for proper operation. The output of the microphone is of the order of milivolts, which is relatively very small in magnitude compared to the precision of the ADC sampling the sensor. This makes it challenging for the microcontroller to detect changes in sensor output. In order to resolve this, a bias and amplifier circuit is designed and implemented to interface the raw sensor output with the MCU. The aim of this circuit is to properly bias the microphone and amplify the sensor output to detect voltage swings caused by sounds. A function generator is connected to the input of the circuit and frequencies are manually swept to test and verify the circuit's frequency response.

\subsection{Memory}

An EEPROM memory chip is interfaced with microcontroller to store cardiac waveforms for future analysis. The microcontroller communicates with the EEPROM chip through a serial peripheral interface (SPI), with the microcontroller as the master and the EEPROM chip as a slave device. Figure 3 depicting the EEPROM interfacing with microcontroller unit using Proteus IDE.

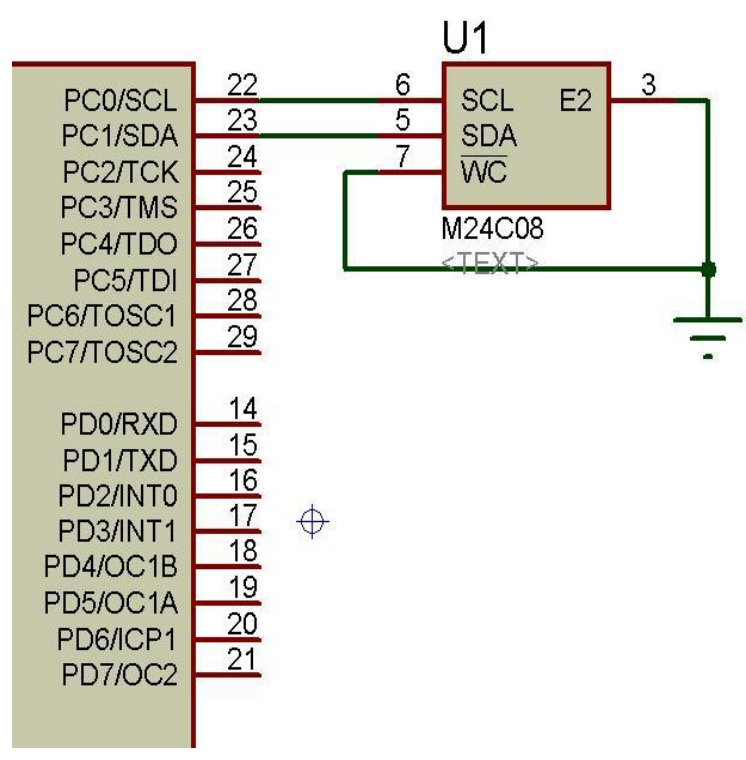

Figure 3. EEPROM interfacing circuit

\subsection{LCD}

A $16 \times 2$ LCD is interfaced with microcontroller to show the heart beats per minute (HBM). A $16 \times 2$ LCD means it can display 16 characters per line and there are two such lines and in this LCD each character is displayed in $5 \times 7$ pixel matrix. In this LCD there are two registers namely, Command and Data. PORT B of microcontroller is connected with the data port of the LCD. Figure 4 showing the LCD interfacing and pin allocation with microcontroller.

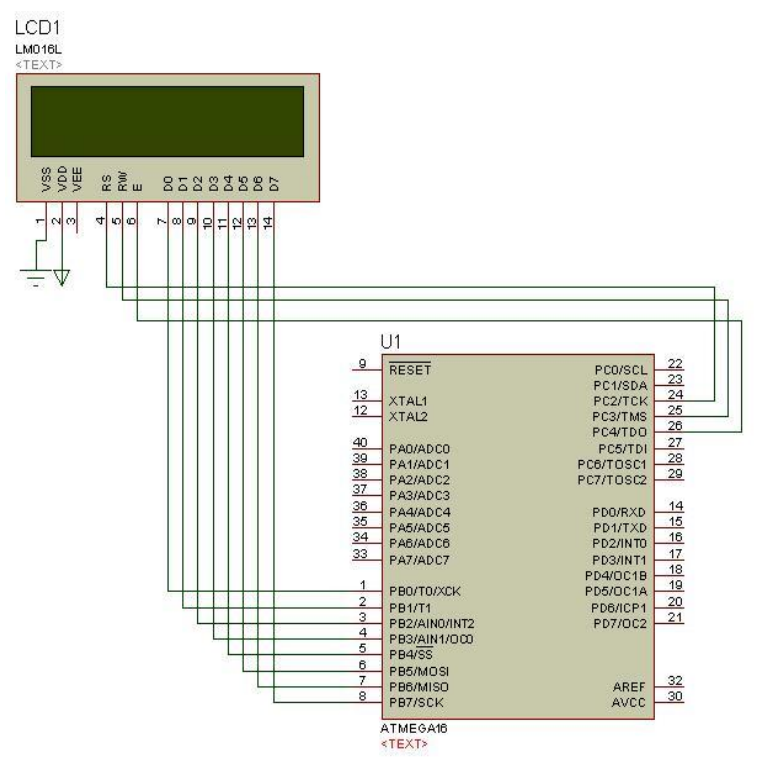

Figure 4. LCD interfacing circuit

\subsection{MATLAB User Interface}

A MATLAB program was developed to read data from the microcontroller through a serial connection with a baud rate of 9600 bits per second and the connection port was periodically queried and read. Received data was then 
processed and displayed on a MATLAB plot in real time. An average of a cardiac waveform was taken to filter out ambient noise and detect when voltage spikes from heartbeats in a clean fashion.

\section{RESULTS}

The results which are obtained from the work done are discussed below-

The analog output which is obtained from stethoscope is divided into two parts:

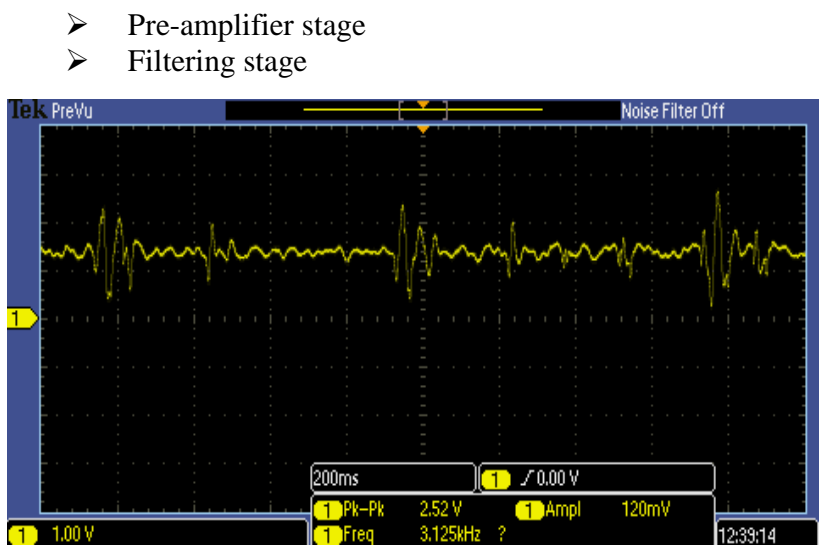

Figure 5. Output of preamplifier stage obtained at the CRO.

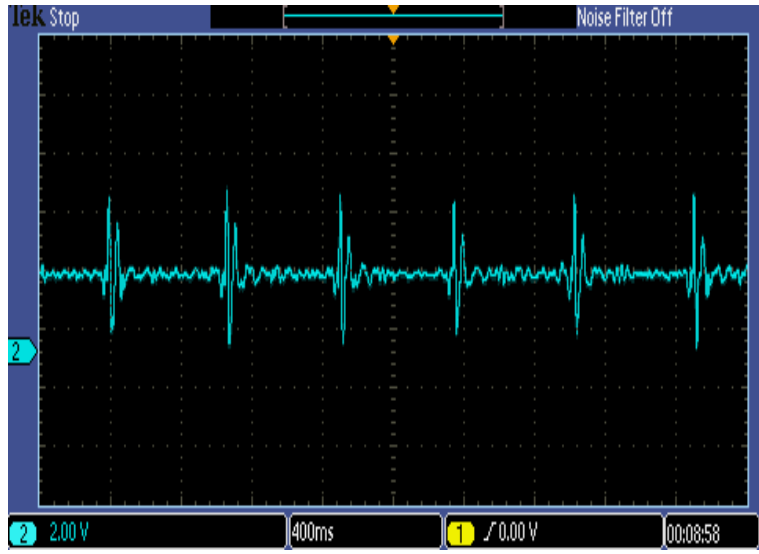

Figure 6. Output obtained after filtering the noise

One of our major objectives is to reduce the noise from the analog input. For this various filtering methodology are being used. Figure 6 shows the output obtained after filtering the noise from the pre-amplifier stage and the results are obtained at CRO.

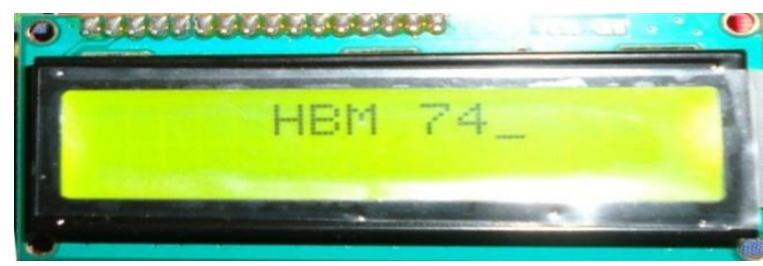

Figure 7. Heart beat rate
Figure 7 shows the heart beat rate obtained from the stethoscope and after counting the number of pulses by microcontroller for 1 minute the result is displayed on LCD.

\section{RESULTS \& CONCLUSION}

This paper presents an electronic stethoscope. This is based on embedded processors and Bluetooth transmission, to meet the requirement of current major clinical application of the heart sound auscultation. There are advantages as follows.

a) The heart sound signal can be amplified, played by speaker in real-time. There is no need of using earphone.

b) The gain of amplification can be modified to satisfy the users' requirement.

c) The LCD screen on the portable device displays heart beats per minute $(\mathrm{HBM})$.

d) The signal can be transmitted to PC by UART interface for MATLAB visualizations and analysis.

\section{REFERENCES}

[1] Yi Luo, "Portable Bluetooth Visual Electrical Stethoscope Research", 11th International Conference on Communication Technology Proceeding (ICCTP) 2008.

[2] Wang; Jian Chen; Yuliang Hu; Zhongwei Jiang; Choi Samjin, "Heart Sound Measurement and Analysis System with Digital Stethoscope," Biomedical Engineering and Informatics, 2009. BMEI '09. 2nd International Conference on, vol., no., pp.1,5, 17-19 Oct. 2009.

[3] El-Bendary, N.; Al-Qaheri, H.; Zawbaa, H.M.; Hamed, M.; ella Hassanien, A.; Qiangfu Zhao; Abraham, A., "HSAS: Heart Sound Authentication System," Nature and Biologically Inspired Computing (NaBIC), 2010 Second World Congress on , vol., no., pp.351,356, 15-17 Dec. 2010

[4] Ji-Yun Shin; Young Cheol Kim; Seung Woon Lim; Eun Jong Cha; Tae-Soo Lee, "Design technology in portable digital esophageal stethoscope," Networked Computing (INC), 2011 The 7th International Conference on , vol., no., pp.94,97, 26-28 Sept. 2011.

[5] Habin Wang, Jian Chen, Choi Samjin, "Heart Sound Measurement And Analysis System with Digital Stethoscope", International Conference on Biomedical Engineering and Informatics, 2009.

[6] Anh Dinh \&Tao Wang, 'Bandage-Size Non-ECG Heart Rate Monitor Using ZigBee Wireless Link',International Conference On Bioinformatics and Biomedical Technology (ICBBT) ,Page 160-163,2010.

[7] Yang Tang, Guitao Cao, Hao Li, "The design of electronic heart sound stethoscope based on Bluetooth" 4th International Conference On Bioinformatics and Biomedical Engineering (ICBBE),Page No. 1-4, 2010.

[8] Jia-Ren Chang Chien, Cheng-Chi Tsi, "The Implementation of a Bluetooth-Based Wireless Phonocardio-Diagnosis System", International Conference an Networking, Sensing and Control,March 2004.

[9] www.atmel.com/Images/doc2466.pdf. 